\title{
Looking Forward, Not Back: Supporting Structuralism in the Present
}

\section{Kerry McKenzie}

This is a close-to-final draft of a paper appearing in Studies in History and Philosophy of Science (Part A), October 2016. Please only cite the final version. Copyright Kerry McKenzie.

\begin{abstract}
The view that the fundamental kind properties are intrinsic properties enjoys reflexive endorsement by most metaphysicians of science. But ontic structural realists deny that there are any fundamental intrinsic properties at all. Given that structuralists distrust intuition as a guide to truth, and given that we currently lack a fundamental physical theory that we could consult instead to order settle the issue, it might seem as if there is simply nowhere for this debate to go at present. However, I will argue that there exists an as-yet untapped resource for arguing for ontic structuralism - namely, the way that fundamentality is conceptualized in our most fundamental physical frameworks. By arguing that physical objects must be subject to the 'Goldilock's principle' if they are to count as fundamental, I argue that we can no longer view the majority of properties defining them as intrinsic. As such, ontic structural realism can be regarded as the right metaphysics for fundamental physics, and that this is so even though we do not yet claim to know precisely what that fundamental physics is.
\end{abstract}

Keywords: Ontic structural realism, quantum field theory, fundamentality, property metaphysics.

\section{Introduction}

Taking Ladyman's seminal 1998 paper to mark its contemporary inception, ontic structural realism (OSR) has now been lurking as a philosophical position for the best part of two decades. Seeming both promising as a response to the master argument against scientific realism and a fitting metaphysic for quantum physics, this period has seen vibrant debate concerning OSR's central contention that it is structure, not objects, 
that is ontologically fundamental. But while those debates have without question been informative and illuminating, several pieces of the structuralist puzzle remain to be put into place. In particular, it seems that structuralists will need to say something about the fundamental kind properties if it is to gain more converts. For if there is one thing that unites the more mainstream metaphysicians of science that OSR stands opposed to, it is the prevailing intuition that the fundamental kind properties are intrinsic in character; fundamental intrinsic properties of any sort, however, are anathema to structuralism. ${ }^{1}$ Of course, structuralists are likely to object at this point that what anyone's intuitions regarding matters of fundamental ontology happen to be are wholly irrelevant to metaphysics. OSR is, after all, an avowedly naturalistic and self-consciously revisionary thesis, and structuralists will hold that we need to look at the relevant physics if we want to develop a defensible metaphysics of it. However, such a move runs into the difficulty that we do not currently take ourselves to know what the truly fundamental kinds are - or at the very least, we do not take ourselves to have a truly fundamental theory of them. Given that we therefore seem to lack the one theory that could be invoked to adjudicate on the matter of what fundamental properties are like, it seems that structuralists must either sit on their hands until we have that fundamental theory, or baldly reject the received intuition; either way, it seems unlikely that they will succeed in persuading the unconverted anytime soon.

In this paper, I want to argue that such pessimism, while understandable, is nevertheless mistaken: OSR need neither be regarded as false, nor as something to be put on ice indefinitely. The reason for this is that, although it is true that we lack a truly fundamental theory of the properties that OSR's sights must be trained on, we do nevertheless possess a framework for thinking about such theories that can plausibly be regarded as fundamental. This is the framework of quantum field theory (QFT). Crucially for structuralism, this framework suggests that the kind properties that will feature in

\footnotetext{
${ }^{1}$ See, e.g. Ladyman and Ross (2007), p. 131: 'talk of unknowable intrinsic natures and individuals is idle and has no justified place in metaphysics... [T] here are objects in our metaphysics but they have been purged of their intrinsic natures, identity, and individuality, and they are not metaphysically fundamental.'
} 
any fundamental theory, whatever it may be, cannot plausibly be regarded as intrinsic. As such, I will argue that a major stumbling block to OSR can be overcome today, and in a thoroughly naturalistic fashion.

In more detail, the layout of my argument will be as follows. In Section 2 I outline what commitment to OSR involves, and emphasize that (a) it is a thesis about the fundamental in particular, and that (b) it seems to prohibit objects from having fundamental intrinsic properties. $^{2}$ In Section 3 I present how the case for OSR is typically made in the literature, and emphasize that the widely-held intuition that the fundamental kind properties are intrinsic has been taken to render OSR unfeasible. In Section 3 I consider how structuralists might get around this problem, given that we currently lack the appropriately fundamental theory of physics that could be appealed to in the hope of settling the issue in their favour: my proposal will be that we can use the framework of QFT to probe such theories prior to our being acquainted with them. In Section 5 I show that the constraints QFT places on fundamental theories means that fundamental kind properties cannot be regarded as intrinsic, on the grounds that the fundamentality of the properties involved has implications for the existence and non-existence of objects distinct from the bearer. Section 6 is the conclusion.

My aim, then, is to show how QFT and the concept of fundamentality embedded within it presents ontic structuralists with a rich new resource - a resource that allows them to deny that fundamental kind properties are intrinsic qua fundamental properties. It will doubtless already be clear, however, that the full articulation of the argument is going to be rather involved, and I should come out and say right at the outset that the argument to be outlined here is at best a suggestive sketch. Its tentativeness owes partly to the fact that there remain a few purely kinematic properties not touched by the argument, and partly to the fact that some relevant mathematical methods required to understand fundamental theories in their full generality await further development. My conclusion will therefore not be a categorical claim that OSR is true, but rather the weaker and more tentative one that everything we know about physics is pointing in that direction.

${ }^{2}$ See though qualifications on this claim in Section 4. 
Nevertheless, this lack of anything definitive to say at this point need not be seen as a criticism: on the contrary, one could interpret it as showing that OSR remains an ongoing, active, and exploratory research programme marching in step with the progress of physics.

\section{OSR as a Fundamentality Thesis}

While it has a number of different articulations, at its core OSR is a proposal concerning what is ontologically fundamental to this world. As the name suggests, the position proposes that the mantle of fundamentality, at least in the actual world, belongs to structure and structure alone. Thus while contemporary structuralists are typically not so radical as to claim that there are no objects simplicter, their proposal is that the category of objects has to be regarded as ontologically secondary to that of structure. To quote Ladyman, they hold that "relational structure is more ontologically fundamental than objects", and for brevity let this be the 'core claim' of OSR. ${ }^{3}$

It is clear that, as a fundamentality thesis, OSR needs to work for the most fundamental objects if it is to work at all. Thus while OSRists have recently elaborated on what OSR has to offer to the special sciences, it must nevertheless be the case that the most fundamental objects of physics are amenable to structuralist analysis if the position is to stand up. In focusing its core claim on the fundamental like this, OSR echoes the overall trend in metaphysics away from theorizing about ordinary objects and towards the task of 'limning fundamental structure' - a project also engaged in by leading analytic metaphysicians of the day, such as Ted Sider, Jonathan Schaffer, and L.A. Paul. But while OSRists take themselves to be distinguished from their more analytic counterparts by their more naturalistic approach, they have been criticized for being less explicit than they as to their conception of ontological priority, and have often been guilty of slipping

${ }^{3}$ Ladyman and Ross op cit., p 145. It might be objected that with this characterization I am equating OSR with its radical version, while OSR also admits a moderate version. But I leave the question of whether the considerations below lend support to one form of structuralism at the expense of the other to another occasion. 
and sliding between non-coextensive relations when spelling out their claims. ${ }^{4}$ Another, perennial criticism of it is that how structural features are supposed to be distinguished from non-structural features is likewise left somewhat imprecise. Clearly, however, without some such distinction we cannot even say of what it is that is to be graced with fundamental status.

In order to clarify OSR's core claim that structure is more fundamental than objects, then, we must clarify both how ontological priority is conceived and also what it is that is meant by 'structure'. So as not to get too bogged down in the details, I suggest that we do the following. With regard to ontological priority, we will follow Chakravartty in using the relation of identity determination to express it: not only is this a relation frequently invoked in the literature, but also one that may be argued on general metaphysical grounds to be an apt relation for structuralism (though I shall not argue for that here). ${ }^{5}$ As such, to the extent that the fundamentality claim definitive of OSR does not go through with respect to identity determination - that is, to the extent that the identities of objects turn out not to be determined by structures - we will take it that it that OSR does not go through simpliciter. Regarding the contrast between structure and non-structure, I propose that we be somewhat liberal do not demand that structuralists must give necessary and sufficient conditions for what counts as an 'object' and what counts as 'structure' before we agree to examine the warrant for their thesis; for plausibly, all that needs to be shown for OSR to go through is that entities ordinarily taken as paradigms of fundamental objects can be shown to be secondary to entities ordinarily taken as paradigms of structures. ${ }^{6}$ With respect to the first category, it seems that there is no better candidate than fundamental particles, and as such our focus will be on those. ${ }^{7}$

4 See e.g. Hawley (2010).

${ }^{5}$ I discuss this further in McKenzie (in preparation).

${ }^{6}$ Part of the reason it seems folly to me to give necessary and sufficient conditions for objecthood is because the concept has evolved so much over the history of physics - a history that is of course itself a large part of the structuralist story.

${ }^{7}$ Note that my argument will go through whether we speak of particles or directly of quantum fields. I should say too that spacetime points are taken as another - perhaps the only other - candidate for 'fundamental object'; but since it is hard to think of what the analogy of fundamental kind properties would be for these entities, it is likely that 
With regard to the second category, we will here follow Maxwell and take structural features to be 'those that are not intrinsic', ${ }^{8}$ where we will understand intrinsic properties somewhat intuitively as that may be possessed by an entity independently of what the rest of the world is like. ${ }^{9}$ As such, intrinsic properties are those properties whose possession neither demands nor precludes the existence of any object distinct from the bearer of the property. Given this characterization of structural features, it follows that relations between and extrinsic properties of objects will qualify as numbering among them. And while one could object that there are intrinsic features of objects that also qualify as structural - compositional structure being the obvious example - it much less clear that fundamental objects could have such features. Our focus here is on just those objects, and we will take it as given that they cannot. ${ }^{10}$

structuralism will be easier to secure in the spacetime case. (See Ladyman and Ross (2007), Section 3.2 for discussion of it.)

8 This quote is from Maxwell (1970), p. 188: while he was an early advocate of epistemic structuralism - but one can find the same equation of structuralism with the denial of intrinsic natures in the OSR canon, e.g. Ladyman and Ross (cf. footnote 1).

9 Thus Weatherson and Marshall (2012) take it that 'a thing has its intrinsic properties in virtue of the way that thing itself, and nothing else, is'; similarly, for Dunn (1990), 'Metaphysically, an intrinsic property of an object is a property that the object has by virtue of itself, depending on no other thing' (p. 178). While the existence of the minor industry in metaphysics dedicated to defining intrinsicality suggests that this could use some sharpening up, I will be content to rest with this informal characterization in what follows. One reason for this is that the by-now standard formal definition of Langton and Lewis (1998) is far from ideal in this context: for one thing, their analysis makes appeal to perfectly natural properties, which for Lewis are both fundamental and intrinsic by definition - precisely that which this paper denies.

10 Afficionados of 'group structural realism' will rightly object at this point that I am simply ignoring the claim that even intrinsic features of fundamental objects may be accounted for structurally - namely, by utilizing the symmetry structures that are so central to contemporary physics theories (see, e.g. Ladyman and Ross Section 3.3). Though regrettably this issue demands a far fuller discussion than I can give it here, suffice to say that - despite once holding this view myself (see e.g. McKenzie 2012) - I no longer think that it works. To see this, it suffices to note that although we know the symmetries of, eg, the Standard Model, many of the kind properties definitive of the particles in it are nevertheless free parameters. Thus symmetry structure does not - at least not in general - determine the fundamental kind properties. (One can at most say 
Putting everything together, then, for us an effort to defend OSR's core claim will consist an effort to show that the identities of fundamental objects are determined exclusively in terms of relations and extrinsic features. An obvious corollary is that no recourse to intrinsic properties will need be made in specifying the identities of fundamental particles.

I propose, then, that if we are to establish OSR then this is what we must try to do. Worryingly for structuralists, however, it has been claimed that this is precisely what we cannot do, for intrinsic properties ineliminably enter into identities at the fundamental level. In particular, has been claimed that the fundamental kind properties that sort particles into species are intrinsic properties, and that these are properties that cannot be left out of the inventory of identifying properties. ${ }^{11}$ In the next section, I will give just enough of an overview of the work that identity determination has done in OSR to bring out the problem of intrinsics. After that, I will consider the difficulties that structuralists face in getting around the problem, before proposing a new and more promising line of attack.

\section{Identity Determination and the Argument from Intrinsics}

One nice feature of the identity -based approach to structuralism is that it applies both in the context of spacetime theory and in quantum mechanics - the two mainstays of philosophy of physics. In the spacetime context, the purported determination of objects by relations is supported by the hole argument in general relativity, since this suggests that primitive identities for spacetime points subjects physics to form of a priori

that they depend on such features; but I no longer regard dependence as a relation of priority. I discuss this a little further in McKenzie (in preparation).) It is however possible that this situation may change with further developments of physics, and indeed many physicists hope that this will be the case. Nevertheless, that observation is clearly of little use in defending structuralism today.

${ }^{11}$ Since kind properties are common both to quantum fields and their associated quanta - the corresponding particles - it doesn't matter for my purposes whether we regard the fundamental objects of physics as particles or fields. I will mostly stick to 'particle'. 
indeterminism. ${ }^{12}$ In the quantum context, the argument is premised on the fact that quantum unlike classical particles cannot in general be distinguished from others of the same type by spatiotemporal location or trajectory. ${ }^{13}$ Furthermore, when such particles become entangled with others, none of the particles in the resulting state can be distinguished from the others through empirical means. Thus unless we are to postulate problematic 'primitive thisnesses' to ground the numerical diversity of entangled particles, all that remains that can play this role are the features of the entanglement relation itself. Fortunately, however, at least in the case of fermions - the basic constituents of matter - one can appeal to such features to perform this role. For in the case of entanglement between two fermions - two electrons, say - the relations may generically be represented as

$$
\frac{1}{\sqrt{2}}\left(\left|\uparrow_{1}\right\rangle\left|\downarrow_{2}\right\rangle-\left|\downarrow_{1}\right\rangle\left|\uparrow_{2}\right\rangle\right)
$$

It is clear that if in this relation we replace a label ' 1 ' with a label ' 2 ', (or vice versa, understanding 'or' as exclusive), the relation disappears, thus establishing that the relation is irreflexive. Since it follows from this that no one object can stand in this relation to itself, we see that there are grounds for saying not only that the particles involved are distinct, but also that it is a feature of the relations between them that underwrites this distinctness. Since facts about an entity's distinctness from other things clearly qualify as facts about its identity, and since (I have claimed) facts about determination of identity should be taken to track facts about priority, these considerations about individuation are often presented as sufficient to secure ontic structuralism about quantum physics. ${ }^{14}$

While these arguments concerning how object identities are determined by structure are central planks of the structuralist scheme, many objections have been pitched against

\footnotetext{
12 See, for example, Ladyman and Ross op. cit., Section 3.2.

13 See, e.g. ibid., Section 3.1.

14 See especially ibid., p. 138. Note that entanglement becomes even more pervasive in quantum field theoretic regimes (see e.g. Lam (2013), particularly Section 2). As such, the conclusions regarding the role of entanglement in securing the diversity of same-kind quantum systems will carry over to the QFT context to be discussed below.
} 
them. For one thing, it is at least strange that while in the spacetime case the argument aims to show there are no haecceities, the analogous argument in the quantum case seems to have them simply ruled out by fiat. 15 For another thing, it is essential to physics that bosons be countable (for example, when confirming quantum field theories via scattering experiments, in which the number of photons produced can be crucial); but the argument, at least as outlined above, seems to give the wrong answer for bosons. ${ }^{16}$ However, the most salient objection for present purposes is that by claiming victory on the basis of these arguments, it seems that OSR aspires only to giving a structural account of the numerical diversity of fundamental particles of a given kind. Such an aspiration clearly takes for granted that particles are sorted into kinds in the first place. But these facts about what makes particles particles of a given kind, as opposed to particles of another kind, seem to constitute just as crucial an aspect of the identities of particles as facts about their distinctness from others of the same type. Clearly, therefore, something needs to be said by the structuralist about their interpretation of fundamental kind properties. Alarmingly for structuralists, however, the view that these properties are intrinsic properties is very much the received view in metaphysics. Since we have identified structural features precisely with 'those that are not intrinsic', this clearly does not bode well. Indeed, things look so bad it would be easy to suspect from the comparative paucity of discussion on this issue in the OSR literature that they have been not so much overlooked as studiously ignored. ${ }^{17}$

Here is how Chakravartty puts the problem.

[L]et us grant for the sake of argument here the orthodox interpretation according

\footnotetext{
15 This is not the case in all structuralists' hands: Steven French, for example, partly premises his structuralism on the fact that haecceities cannot be ruled out a priori (see e.g. French 1998). But French is an exception here, especially now that James Ladyman no longer bases his structuralism on the underdetermination argument.

16 At least as I understand the presentation in Saunders (2003); see Huggett and Norton (2014) for a much fuller discussion.

17 Here again I emphasize, as I did at footnote 10, that structuralists have had things to say about kind properties. But it is certainly the case that structuralists have sometimes spoken as if the above considerations regarding diversity are in themselves sufficient to establish the position, and Chakravartty is right to protest that that is much too hasty.
} 
to which quantum particles have relations that cannot be analyzed in terms of anything intrinsic. Would this demonstrate that the identities of these objects are extrinsic, in the manner suggested by the ontological priority thesis of noneliminative OSR? ${ }^{18}$

The answer, I suggest, is no. In order for the appeal to extrinsic [properties] in this context to offer any support to non-eliminative OSR, it would seem that one of the two following conditions should obtain. Either it should be the case that not just some, but all properties of the particles described by quantum theory are extrinsic, or it should be the case that whatever intrinsic properties the theory does attribute to them do not determine their identity. [...] But neither condition obtains. While some properties are described by quantum theory in terms of relations of entanglement, others are not. Mass and charge, for example, are stateindependent intrinsic properties of subatomic particles, whose attribution thus violates the first condition that all properties be extrinsic... [T] he fact remains that wherever one applies the concept of a particle, the theory presents descriptions of what appear to be intrinsic properties which are constitutive of their identity. ${ }^{19}$

The view that Chakravartty reports in this passage is certainly not idiosyncratic: it is so well-entrenched that it probably deserves to be called the 'received view' in metaphysics. His fellow dispositional essentialist Bird, for example, states that 'no-one has suggested that charge, rest mass and spin are not intrinsic', and indeed the same policy on fundamental properties is shared even by their Humean rivals (most obviously, Lewis). ${ }^{20}$ But if metaphysicians as divided on virtually every other issue of scientific metaphysics can nevertheless agree on the issue of intrinsicality, then structuralists had better give an impressive argument as to why the view is wrong. Let this objection that the intrinsicality of these properties forecloses OSR be the 'argument from intrinsics'. Since OSR is the thesis that no identity-determining features of fundamental objects are

${ }^{18}$ By 'non-eliminative OSR', Chakravartty means any form of OSR in which objects are taken to exist but taken to be non-fundamental

${ }^{19}$ Chakravartty (2012), p. 204.

${ }^{20}$ Bird 2007, p. 125; e.g. Lewis 1983, p. 357. 
intrinsic in character, the argument from intrinsics throws the gauntlet firmly at the structuralists' feet.

\section{The Structuralist Dilemma and A New Strategy}

Structuralists therefore need to find a way of rebutting the argument from intrinsics. What, then, are they to say? No doubt, structuralists will be quick to point out that while it is certainly the case that kind properties like charge and mass are 'commonly regarded'21 as intrinsic - while it may even be that 'no-one has suggested' that they are not intrinsic, at least no-one failing to self-identify as a structuralist - remarkably little argument for this claim is ever given. ${ }^{22}$ But if the intrinsic properties are those that can be had even by a lonely object, then given that we will obviously never encounter such objects it is just very hard to say whether these properties are intrinsic or not - in much the same way that it is difficult, as Mach noted, to say what would happen to a bucket in an empty universe. ${ }^{23}$ Given this, rather than simply backing down before the intuitions that metaphysicians may happen to share on the matter, the structuralist will likely insist that what we must do - indeed, all we can do - is look at what a suitable theory of physics has to say about it, and defer to that.

But if that's the plan, then structuralists face a problem. This problem is that, as already underlined, OSR is ultimately a fundamentality thesis, but we do not take ourselves to possess a truly fundamental theory. As such, we do not take ourselves to have a theory that would definitely answer the question of whether or not we can regard the fundamental kind properties as intrinsic or not. Thus even the Standard Model of particle physics (describing the interactions of quarks, leptons, and gauge bosons), while without doubt the most fundamental theory we have been able to produce to date, is nevertheless

\footnotetext{
21 Cf. Esfeld 2014.

22 The closest one seems to get is the argument from dispositional that their postulated intrinsicality is not inconsistent with their assumed dispositional nature. See e.g. Bird $o p$ cit.

${ }^{23}$ Some of the difficulties involved in justifying the claim that the properties of physical objects are intrinsic are given in Slater and Haufe (2009), Section 4.
} 
not thought not to be truly fundamental - partly on account of its failure to incorporate gravity, its surfeit of free parameters, and suspected divergence in the high-energy limit. As such, it cannot definitively answer the question of whether the fundamental properties are intrinsic; and if it isn't qualified then it is hard to see what is. Given this, it seems that structuralists must simply sit on their hands until a truly fundamental theory comes along that they can cite in support of their argument. But that may be a long time in coming - assuming it will ever happen at all.

The defence of OSR at this point therefore seems to have run into the ground. While structuralists face an uphill struggle in talking metaphysicians out of an intuition, apparently universally shared, concerning fundamental kinds, they want for the theory that they can appeal to in order to try and settle the issue in their favour. What I will propose here, however, is that contrary to appearances structuralists need not simply down tools for now, for there may be a third way of defending OSR that relies neither on intuition trading nor on our possession of a truly fundamental theory. What makes this third way possible is that, while we lack such a theory, we do nevertheless have a framework for theories that may well be fundamental. This is the framework of quantum field theory. ${ }^{24}$ What it means to call QFT a framework and not so much a theory (despite the name) is that, when we think of a theory in physics, we think of a set of objects, such as fields and their associated particles, and a law of their interaction; QFT, on the other hand, constitutes a set of principles that constrain any theory that claims to describe relativistic quantum systems. ${ }^{25}$ As such, this framework can be used to define an unlimited number of different theories, depending on the fields whose behaviour we want to model and the interactions we postulate between them. The crucial claim that I will make here is that when we reflect upon the constraints that the QFT framework places on fundamental theories in particular, we see that there is actually positive reason to deny that the fundamental kind properties are intrinsic - at least in the vast majority

${ }^{24}$ On the notion of theories as contrasted with frameworks for theories, see Shimony (1987), p. 209.

${ }^{25}$ For example, that any law must be Lorentz invariant, that the states of QFT systems must superpose, and so on. 
of cases. ${ }^{26}$ This is because their very status as fundamental kind properties turns out to be highly sensitive to what other kinds exist in the world besides the kind in question. As a result, we should regard the fundamental level as populated not by fundamental objects with intrinsic natures, but rather with objects whose very status as fundamental enmeshes them in a rich nexus of dependences to one another. It should be noted, then, that since my claim is that the fundamental kind properties are extrinsic qua fundamental properties, the argument has nothing to say about worlds in which those kinds exist but are not fundamental - on the (highly) questionable supposition that such worlds are possible. But since it is giving an account of the fundamental in particular that OSR is concerned with, the fact that these properties cannot be regarded as intrinsic wherever they occur as fundamental properties should be good enough for it.

In the next section of this paper I will try to make good on my claim that fundamentality conceptualized à la QFT offers a new line of support to structuralism. Before I do that, however, I need to make a few qualifications and disclaimers. The first of these qualifications is that, as will already have been noticed, I have already slipped in that my argument, although highly general, will apply only to 'the vast majority' of fundamental properties. The reason for this qualification is that, while any particle in QFT must have some value of spin and parity (as a consequence of the symmetries of Minkowski spacetime), my argument will not apply to these properties. This is because my argument applies only to those properties that are associated with a coupling parameter in the Lagrangian defining the theory: these are any properties through which particles interact with other particles, as well as the property of mass. ${ }^{27}$ The value that the parameter takes determines the strength of the interactions involved or the effective mass of the particle respectively. ${ }^{28}$ Spin and parity, on the other hand, are not associated with coupling parameters, and while there is reason to think that these are the only

26 OSRists of course needs it to be the case that no fundamental property is intrinsic, and we shall see my argument does not apply to quite all. More on this to follow presently.

27 We can think of the Lagrangian as just another way of expressing the law of nature referred to by the theory.

${ }^{28}$ Mass has this status even in anticipation of a quantum theory of gravitation because it may in a sense be regarded, in the QFT context, as encoding the interactions of a field with itself. See e.g. Susskind (2012) for a qualitative description. 
properties that are not touched by the argument I will not hazard here what OSR should say about them. Nevertheless, it remains that my argument will see off the vast majority of the properties cited by OSR's critics, clearing the way for us to focus on these last aspects. ${ }^{29}$

A further limitation to point out is that my argument will not be conclusive even with respect to those properties that it succeeds in applying to. The reason is that, in the approach I propose, the fundamentality of properties is to be conceived of in terms of the fundamentality of the theories in which they occur; but precisely what features theories must have to qualify as fundamental is something not yet understood in its full generality. My argument will apply only to the class of fundamental theories that we do understand namely, the asymptotically free theories. But such theories are only a subset of the class of asymptotically safe theories - where 'asymptotically safe' is just another word for fundamental QFTs. While the features of asymptotically free theories are rather wellunderstood, the general features of the remaining theories are not known at present indeed they are difficult even 'to imagine or investigate'. ${ }^{30}$ It is thus understandable that my argument focuses only on the former case, but this strategy obviously runs the risk that my conclusions will not hold when we generalize out. However, the reason that the features of the more general class are not known is because the interrelationships between the couplings in these theories are so involved and so complex that theorizing about them proves mathematically prohibitive, at least by methods currently to hand. It would thus in my opinion be very surprising if the holism that I will claim is manifest in the restricted class were not present in the more general one - indeed surprising if not still more pronounced. Nevertheless, for the moment that remains mere speculation on my part.

${ }^{29}$ I might point out too that by focusing primarily on interaction properties I am in no way begging the question, for - as dispositional essentialists have long been at pains to point out - the mere fact that a property is an interaction property does nothing to undermine its intrinsic status. I return to this point below.

30 Wilczek (1999), p. 2; Kang (1975), p. 11. 
A final point to underline at this stage is that my argument is going to assume that QFT is indeed a fundamental framework for theories, so that we have reason to think that the fundamental level can indeed be described within it. What is certain is that QFT is our most fundamental framework for physics at present, at least that we are able to submit to empirical test; there is moreover increasing optimism about it being a truly fundamental framework, owing to a growing sense that it is only technical obstacles that stand in the way of a quantum field theoretic treatment of gravity. ${ }^{31}$ Nevertheless, since my argument depends on spacetime having the continuous structure that it has in QFT, and since some (speculative) new physics frameworks deny this, the truth of my conclusions is vulnerable to change as physics develops. Fundamentality is in that sense a highly theoretical concept. However, I trust that those of a naturalistic outlook will not see this as necessarily a criticism, as opposed to simply part of what it means to do naturalistic metaphysics.

With those qualifications and clarifications in place, let me proceed to the argument.

\section{The Strategy Implemented: Supporting Structuralism in the Present via Fundamentality Constraints in QFT}

My aim, then is to show that the kind properties defining the fundamental particles of physics are not intrinsic, and - to repeat - the properties I am focusing on are the property of mass and the properties through which objects interact: let us call the latter the 'interaction properties'. ${ }^{32}$ Having already said a little about what I'll intend by 'intrinsic', it remains to say something about how 'fundamental kind property' should be understood.

Since the aim of the game here is to show that the properties defining fundamental objects are not intrinsic, it seems natural (were it not so already) to define 'fundamental kind property' as one of the properties that sort the fundamental objects into kinds. But

31 On this, see Percacci 2009.

32 The most fundamental interaction properties we know of are charge, weak isospin and colour charge, but it is likely that at some of these are not truly fundamental. 
doing so just shifts the problem of defining 'fundamental kind property' to defining 'fundamental objects', and in any relativistic quantum theory - that is, in any quantum field theory - this is notoriously difficult to do. In particular, given that in these theories even (what are regarded as) point particles can decay into other particles, any attempt to define fundamentality in mereological terms seems a hopeless enterprise. Partly as a result of this, it is now standard to regard a fundamental particle as one whose field appears directly in a fundamental Lagrangian - that is, a particle whose interactions are specified by a fundamental law of nature. ${ }^{33}$ We should therefore regard the fundamental kind properties as the properties that sort the objects evolving in accordance with the fundamental laws of nature into kinds. In so doing, we use the fundamental laws to define the fundamental kind properties.

To find out whether these properties are intrinsic, then, it appears that we must consult the fundamental laws of physics, look at the fields and particles in them, and see what the theory has to say about their metaphysical features (of course in tandem with some appropriate metaphysical theory). But if that is the case, then it seems that we are back at the problem we started with - namely, our lack of fundamental theory. However, to draw this conclusion would be overlook the fact that while we may lack knowledge of the fundamental laws of nature, we can hazard that they accord with quantum field theory. As I will argue, this fact allows us to identify generic features that any fundamental law must satisfy, making it possible to make substantive metaphysical claims concerning fundamental properties, even though we remain in the dark right now as to what those properties are. ${ }^{34}$

Let us then turn to those generic features. The simple but crucial insight is that, in the QFT framework, to call a law fundamental is to make a quite specific predication of it: it is to affirm, at the very least, that the law stays mathematically consistent in the limit of infinite energy. We do after all take probing structures at higher and higher energies - or, equivalently, shorter and shorter distances - to correspond to probing more and more fundamental regimes, and the continuity of space presupposed in Minkowski spacetime

33 See, e.g. Heisenberg (1975); Weinberg (1996).

34 Modulo the caveat regarding kinematical properties made above. 
entails that the energy range that is available to be probed is in principle infinite. We might thus follow Huggett and Weingard in saying that theories valid in the infinite energy limit are 'continuum compatible'. ${ }^{35}$ But although this condition on a fundamental law is easy to state, stating what laws must be like to actually satisfy it is a much more difficult endeavor, assuming that the laws describe any interactions. ${ }^{36}$ What we do know is that continuum compatibility is an extraordinarily stringent requirement - so much so that there is a general feeling in contemporary particle physics that if we can find $a$ theory of fundamental entities that satisfies it, then we have found the theory, and so an actual fundamental law of nature. Part of the reason it is so hard to write down laws satisfying continuum compatibility is that the coupling parameters that appear in QFT Lagrangians - to repeat, those associated with the masses of particles and the interactions between them - change their values as the energy is scaled up, in a way described by the renormalization group equation. ${ }^{37}$ Thus results in the properties whose magnitudes are given by the values of these couplings - such as the particle's mass and charge - becoming likewise scale-dependent. ${ }^{38}$ Since generically we can expect functions to diverge as their arguments do, we can expect the mass or strength of interactions of particles in an arbitrary quantum field theory to likewise diverge in the limit - making the theory that they occur in mathematically ill-defined too. Thus a minimal consistency condition on any fundamental QFT is that all of its coupling parameters stay finite in the infinite-energy limit. Those theories for which all couplings remain finite are said to be 'asymptotically safe'. Theories for which all of the couplings not only stay finite but also have the special feature of tending to zero in the limit are known as theories that are 'asymptotically free'.

\footnotetext{
${ }^{35}$ Huggett and Weingard (1995).

${ }^{36}$ I will consider how the existence of free theories impacts upon my conclusions below. ${ }^{37}$ See e.g. Ryder (1996), p. 325.

38 Thus, for example, the charge on an electron increases with energy, and the mass of a quark decreases. Thus what we refer to as 'the' charge on an electron is really just the value of the coupling constant measured in e.g Thomson scattering experiments at very low energy. See e.g. Maggiore (2009), Section 5.9.
} 
As already mentioned, however, the only theories we are able to get a grip on at the moment are those that are asymptotically free. While the lack of generality is unfortunate, there is nevertheless now a great deal that we can say about the asymptotically free case. The most important thing we know is that any asymptotically free theory has to be a renormalizable local gauge theory: that is, it must have a form that it is invariant under the transformations of a gauge symmetry group, and contains no non-renormalizable terms. ${ }^{39}$ For present purposes, we do not need to know precisely what these terms mean: however, what we do need to appreciate is that - and somewhat amazingly - this requirement gives us a recipe for uniquely pinning down the laws of nature. In a little more detail, it gives us a recipe for pinning down the laws of interaction pertaining to fundamental constituents of matter - that is, the fundamental fermions given a specification of what these fermions are. For example, suppose we are handed 6 quarks - that is, particles that carry colour in addition to some determinate mass and spin. Then our knowledge that the law appropriate to these particles must have a local gauge symmetry if it is to be fundamental determines what the form of that law is: a renormalizable theory with gauge group $\mathrm{SU}(3)$ is the unique theory with this property. ${ }^{40}$ Crucially, armed with this information one can go on and show that, in addition to the kinematic terms describing the quarks' free motion, the law must contain a term describing the quarks interacting with a specific set of bosons through a coupling constant, $g$. More generally, whenever we ascribe fundamental interaction properties to fermions, the requirement of local gauge invariance implies that they couple through those properties to a set of bosons, with the strength of the coupling measured by a coupling constant featuring in the relevant Lagrangian.

${ }^{39}$ See e.g. Wilczek op cit.. It must furthermore be a non-Abelian gauge theory, which means that the bosons will carry the same charges through which the fermions interact with each other and with the bosons.

${ }^{40}$ Here I assume that quark colour states have the structure, in all possible worlds, of the fundamental representation of SU3. The fact that the canonical discussions of the metaphysics of properties have focused more or less exclusively on classical properties means that I cannot simply cite some authoritative figure in support of this claim. For now, suffice to say that I cannot imagine how someone could deny it; nor does it beg the question at hand. 
This requirement that asymptotically free theories have to be local gauge theories has profound implications for the possibility that we may actually discover the fundamental laws of nature, given empirical information concerning what fundamental kinds there are. But it has equally profound implications for the intrinsicality of the properties of the particles that feature in those theories. These implications may be divided into two classes: firstly, those concerning the relationship of fermions to bosons; secondly, those concerning the connections between all types of particles with (other) fermions. The first class of implications is easy to state: it is that any interaction property had by a fundamental fermion cannot be regarded as intrinsic, because the possession of any such property requires the existence of bosons. That is, since fundamental particles are particles whose fields appear in fundamental Lagrangians, and since the laws of interaction for fundamental fermions require the existence of gauge bosons, those bosons are needed in order for there to be fermions that we can designate as fundamental at all. ${ }^{41}$ Thus it cannot be said that the fundamental interaction properties partly definitive of fundamental fermions are intrinsic in character: on the contrary, their very status as fundamental brings in its train entities distinct from themselves.

On account of the relationship that fermions with these properties must stand in to the corresponding gauge bosons if they are to be fundamental, then, we are no longer able to regard many of the properties of fundamental fermions as intrinsic - in particular, the interaction properties. However, this is not the only reason why considerations of fundamentality undermine the intrinsicality of the features defining fundamental particles. A further reason is that, while local gauge invariance is a necessary feature of any fundamental law, it is in itself insufficient: for if there are too many types of fermions included in the law then the fundamentality of that law is lost. The reason behind this is

\footnotetext{
41 Steven French and I made the argument that interaction properties cannot be intrinsic because of their relation to gauge bosons in French and McKenzie (2012). That argument was criticized by Livanios however, on the grounds that this relation could be contingent (see Livanios 2012, p. 32). This argument of the present paper improves on that argument, in that it exploits the fact that we are not considering charge simpliciter, but charge qua fundamental property. It is the extra constraints placed on properties featuring in fundamental theories in particular that links charge to gauge bosons.
} 
that the behavior of the couplings in a local gauge theory is sensitive to how many kinds (or 'flavours') of fermions exist in the theory, in such a way that (at least some of) the couplings will diverge if there are too many of them. ${ }^{42}$ For example, in the case of a theory with a local SU3 gauge symmetry, such as QCD, the number of quark types in that theory must be no greater than 16 the theory is to be valid to arbitrarily high energy; otherwise the coupling associated with colour will diverge in the limit, and the law with it. ${ }^{43}$ And, to repeat, since a fundamental particle is one whose field appears in a fundamental Lagrangian, it follows that we cannot say that the fundamental interaction properties of any given kind are intrinsic, because their very status as fundamental kind properties depends on there not being too many other kinds in the world besides the kind in question. Moreover, while a less obvious route to intrinsicality than the last, this approach is more extensive in its reach. This is for at least two reasons. For one thing, the argument applies just as much to the properties of bosons as to those of fermions: the colour charge on a gluon, say, will cease to be capable of being regarded as fundamental if there are 17 or more fermion types inhabiting the world alongside the gluon, just as the colour charge on any given type of fermion will suffer the same fate. But furthermore, the argument has implications for our ability to regard the property of mass as intrinsic in addition to the interaction properties. This is because the masses of particles in QFT are also functions of the energy, and the renormalization group equation describing the coupling parameter corresponding to mass is a function of the all couplings present in the theory. As a result of this, if the couplings diverge we can expect the mass to diverge as well. So for broadly the same reasons as before, it seems that we cannot regard particle masses as intrinsic in any straightforward sense either: we simply cannot postulate fundamental particles and predicate mass of them without keeping an eye on what other

42 Thus, as Wilczek puts it, 'One finds that only nonabelian gauge theories with simple matter content, and no non-renormalizable couplings, satisfy this criterion (op cit., p. 3); similarly for Ho-Kim and Yem, 'theories of non-Abelian gauge fields and fermion multiplets are asymptotically free only if the theory does not have too many fermions' (1998, p. 278). (p. 535). 
particles inhabit the world alongside the particle. As such, we cannot say that mass is both a fundamental kind property and intrinsic at the same time.

We have seen, then, that given how fundamentality is conceived of in physics we cannot consider either interaction properties or the property of mass as intrinsic in anything like the usual sense. Without the gauge bosons to supplement the fundamental fermions, the interaction properties of the latter could not qualify as fundamental; too many fermions in the picture, on the other hand, will rob all particles of fundamental status. What we have arrived at, then, is a sort of 'Goldilock's principle' governing the fundamental particle kinds: a kind can only qualify as fundamental if there are other kinds inhabiting the world alongside it, so long as there are not too many. ${ }^{4}$ The fundamental level of the world, then, seems not made up of entities with their own 'intrinsic natures': each must be sensitive to the totality of what else exists in order to be fundamental at all.

It might be objected at this point that all this has been derived from the assumption that the fundamental kinds partake in laws of interaction. For it is interactions in particular that make it difficult to define laws that stay well-defined in the limits appropriate to fundamental laws, and which result in the constraints on matter content expressed by the Goldilock's principle. ${ }^{45}$ But one could object that the fundamental kinds that we find in this world can all feature in laws without interactions - specifically, the laws of free motion to which interaction terms are added when interaction laws are written down. As such, one could argue that although the quarks (for example) that feature in our world might need the relevant gauge bosons in order to interact, they could quite well exist without them - namely, in worlds in which they undergo no interaction at all. And since

${ }^{43}$ One can see this from looking at the renormalization equation governing the colour coupling: see for example Ho-Kim and Yem op cit., equation 15.83 (p. 535).

${ }^{44}$ I owe this nice phrase to David Brink.

45 Note that the supposition that the fields involved are interacting does not in itself tell against intrinsicality. For quantum field theory admits laws containing only a single field interacting with itself - although, by the above arguments, we know that no such law can be regarded as fundamental. 
there are worlds containing only lone quarks, albeit of necessity free quarks, it must be possible to regard the properties of fundamental objects as intrinsic after all. ${ }^{46}$

However, it seems to me that this objection does not succeed, for at least a couple of reasons. First of all, we know that the fundamental objects in our world are as a matter of fact interacting - otherwise we could never know about them (indeed we ourselves wouldn't exist at all). And we know from the fact that they are interacting that some of their properties are scale-dependent. It strikes me that an object in a world without interactions - and as such an object whose properties are not scale-dependent - cannot reasonably be said to count as a duplicate of an object whose properties are scaledependent in the way that actual-world objects are. For this reason - and although doing so is commonplace in philosophy of physics - I think we must be extremely cautious about drawing conclusions about the metaphysics of actual-worldly objects based on claims about worlds described by free theories. ${ }^{47}$ But in any case, the (putative) fact that an object could exist without some other object, such as a gauge boson, existing is not sufficient to make its properties intrinsic: it must in addition be the case that its possessing those properties does not preclude the existence of other objects alongside it. But we know that the presence of fundamental objects with colour prohibits the existence of too many fermions in any worlds in which those objects interact. And it seems to me that the fact that there are prohibitions on what can exist alongside coloured objects in even some subset of the worlds in which they exist - namely the set of worlds with interactions - is incompatible with the supposition of its intrinsicality.

In sum, then, we may say that contra Chakravartty, Bird, and seemingly almost every other metaphysician of science outwith structuralism, the fundamental kind properties cannot be regarded as intrinsic after all. Insofar as OSR is the thesis that fundamental objects lack intrinsic properties, then, it is left standing tall.

\section{Conclusion}

46 This objection has likewise been raised by Livanios op cit., pp. 33-4.

${ }^{47}$ On this point, see also Baker 2013, Section 3. 
The main argument of this paper has been that the concept of fundamentality as understood in QFT offers a rich new resource for defenders of ontic structuralism. It is a resource that allows structuralists to attack the argument from intrinsics in advance of the truly fundamental theory that, prima facie, one would have thought would be needed to deny the force of their opponents' intuitions. However, as I have taken pains to note, the argument is not yet conclusive. For one thing, I have considered only the class of fundamental quantum field theories that are well-understood at present - namely, the asymptotically free theories - and while these considerations surely count at least as evidence for the conclusions I have drawn, it nevertheless may well be that the fundamental level is not described by a theory in this class. For all that I have speculated that similar - perhaps yet more exaggerated - conclusions will hold in the more general case, that remains a speculation on my part. Another limitation is that there seem to be properties that are not touched by my argument, namely the purely kinematic property of spin and parity. Most significantly of all, perhaps, I have proceeded as QFT is a fundamental framework; but QFT's capacity to incorporate gravity very much remains to be seen.

Nevertheless, it seems clear that the arguments of the last section are strongly supportive of ontic structuralism and its denial of fundamental intrinsic properties. I therefore hope to have shown that the concept of fundamentality itself presages a structuralist future, even though we still look forward to discovering what exactly the fundamental constituents of the world are.

\section{References}

Baker, David (2013). 'Identity, Superselection Theory, and the Statistical Properties of Quantum Fields', Philosophy of Science, Vol. 80, No. 2, April 2013, pp. 262-85.

Bird, Alexander (2007). Nature's Metaphysics. Oxford: Oxford University Press. 
Chakravartty, Anjan (2012). 'Ontological Priority: The Conceptual Basis of NonEliminative, Ontic Structural Realism', in E. M. Landry \& D. P. Rickles (eds.), Structural Realism: Structure, Object, and Causality, Western Ontario Series in Philosophy of Science, Springer.

Dunn, J.M. (1990). 'Relevant Predication 2: Intrinsic Properties and Internal Relations', Philosophical Studies 60: 177-206.

Esfeld, Michael (2004). 'Quantum entanglement and a metaphysics of relations', Studies in History and Philosophy of Modern Physics 35: pp. 601-17.

Esfeld, Michael 2014: 'Quantum Humeanism, or: physicalism without properties', Philosophical Quarterly 64 (256): 453-470.

Ho-Kim, Quang and Pham Xuan Yem (1998). Elementary Particles and their Interactions, Springer.

Huggett, Nick and Robert Weingard (1996). 'Exposing the Machinery of Infinite Renormalization', Philosophy of Science 63 (3): 167.

French, Steven (2006). 'Structure as a Weapon of the Realist', Proceedings of the Aristotelian Society, Volume 106, Issue 1, pp. 170-187, June 2006.

French, Steven (2010). 'The Interdependence of Objects, Structure and Dependence', Synthese, 175: 89-109.

French, Steven (1998). 'On the Withering Away of Physical Objects', in Castellani, Elena (ed.) (1998): Interpreting Bodies: Classical and Quantum Objects in Modern Physics, Princeton University Press, Princeton, pp. 93-113.

French, S. and Kerry McKenzie (2012). 'Thinking Outside the Toolbox: Toward a more Productive Engagement between Metaphysics and Philosophy of Physics', European Journal of Analytic Philosophy 8 (1): 42-59.

Hawley, Katherine (2010). 'Throwing the Baby Out with the Bathwater: Critical notice of 
Every Thing Must Go by Ladyman, Ross et al.', Metascience, 19.2 (July 2010), pp. 174-9.

Heisenberg, Werner (1975): 'Development of concepts in the history of quantum theory', American Journal of Physics 43 (5): 389-394.

Huggett, N. and J. Norton (2014). 'Weak Discernability for Quanta, the Right Way', British Journal for the Philosophy of Science, 65 (1): 39-58.

Kang, J.S. (1975). 'Representation Constraint on Asymptotic Freedom in the High Symmetry Limit', Physical Review D11, 1563.

Kantorovich, Aharon (2003). 'The priority of internal symmetries in particle physics', Studies In History and Philosophy of Science Part B: Studies In History and Philosophy of Modern Physics, Volume 34, Issue 4, December 2003, pp. 651-675.

Ladyman, James (2014). 'Structural Realism', The Stanford Encyclopedia of Philosophy (Spring 2014 Edition), Edward N. Zalta (ed.), URL = <http://plato.stanford.edu/archives/spr2014/entries/structural-realism/>.

Ladyman, James and Don Ross 2007. Every Thing Must Go. Oxford University Press. Oxford: UK.

Lam, Vincent 2013. 'The Entanglement Structure of Quantum Field Systems', International Studies in Philosophy of Science, 27 (1), 59-72.

Langton, Rae and David Lewis (1998). 'Defining “Intrinsic"', in Philosophy and Phenomenological Research, 58 (2): 333-45.

Lewis, D. 1983. 'New Work for a Theory of Universals', Australasian Journal of Philosophy 61: 343-377. 
Livanios, Vassilios (2013). 'Is There a (Compelling) Gauge-Theoretic Argument against the Intrinsicality of Fundamental Properties?', European Journal of Analytic Philosophy 8.2: $30-38$.

Maxwell, Grover (1970). ' 'Structural realism and the meaning of theoretical terms', in Analyses of Theories and Methods of Physics and Physiology, volume IV of Minnesota Studies in the Philosophy of Science, pages 181-192. Minneapolis: University of Minnesota Press.

Maggiore, Michele (2009): A Modern Introduction to Quantum Field Theory, Oxford: Oxford University Press.

McKenzie, K. 2013. 'Priority and Particle Physics: Ontic Structural Realism as a Priority Thesis', British Journal for the Philosophy of Science, doi:10.1093/bjps/axt017

McKenzie, K (in preparation): The Plurality of Priority.

Nounou, Antigone M. (2012). 'Kinds of Objects and Varieties of Properties', in E. M. Landry \& D. P. Rickles (eds.), Structural Realism: Structure, Object, and Causality, Western Ontario Series in Philosophy of Science, Springer, pp. 117-33.

Paul, L.A. 2012. 'Metaphysics as modeling: the handmaiden's tale', Philosophical Studies 160:1-29.

Percacci, Roberto (2009): 'The Asymptotic Safety FAQs', webpage, URL = http://www.percacci.it/roberto/physics/as/faq.html

Saunders, Simon (2003). 'Physics and Leibniz's principles', in K. Brading and E. Castellani, (eds.), Symmetries in Physics: Philosophical Reflections, Cambridge: Cambridge University Press.

Shimony, Abner (1987): 'The methodology of synthesis: parts and wholes in low-energy physics,' Chapter 14 of Search for a Naturalistic World View, Volume 2 of Natural Science and Metaphysics, Cambridge University Press (1993). 
Sider, Theodore (2011): Writing the Book of the World. Oxford: Oxford University Press.

Schaffer, Jonathan (2009): 'On What Grounds What', in Metametaphysics: New Essays on the Foundations of Ontology, eds. David Manley, David J. Chalmers \& Ryan Wasserman (eds.); Oxford University Press.

Slater, Matthew H. and Chris Haufe (2009): 'Where No Mind has Gone Before', International Studies in the Philosophy of Science 23(3): 265-276.

Susskind, Leon (2012). 'Supersymmetry and Grand Unification' (Lecture 1), available at https://www.youtube.com/watch?v=W6srShxBCrk.

Weatherson, Brian and Marshall, Dan (2014). 'Intrinsic vs. Extrinsic Properties', The Stanford Encyclopedia of Philosophy (Fall 2014 Edition), Edward N. Zalta (ed.), URL = http://plato.stanford.edu/archives/fall2014/entries/intrinsic-extrinsic/

Weinberg, Steven (1996): 'What is an Elementary Particle?', Beam Line, Spring 1997, Vol. 27, No. 1, pp. 17-21

Wilczek, Frank (1997). 'What QCD Tells us about Nature - and Why We Should Listen', available at arXiv:hep-ph/9907340. 